\title{
A nuclear-encoded chloroplast protein harboring a single CRM domain plays an important role in the Arabidopsis growth and stress response
}

\author{
Kwanuk Lee ${ }^{1}$, Hwa Jung Lee ${ }^{1}$, Dong Hyun Kim ${ }^{1}$, Young Jeon ${ }^{2}$, Hyun-Sook Pai ${ }^{2}$ and Hunseung Kang ${ }^{1 *}$
}

\begin{abstract}
Background: Although several chloroplast RNA splicing and ribosome maturation (CRM) domain-containing proteins have been characterized for intron splicing and rRNA processing during chloroplast gene expression, the functional role of a majority of CRM domain proteins in plant growth and development as well as chloroplast RNA metabolism remains largely unknown. Here, we characterized the developmental and stress response roles of a nuclear-encoded chloroplast protein harboring a single CRM domain (At4g39040), designated CFM4, in Arabidopsis thaliana.

Results: Analysis of CFM4-GFP fusion proteins revealed that CFM4 is localized to chloroplasts. The loss-of-function T-DNA insertion mutants for CFM4 (cfm4) displayed retarded growth and delayed senescence, suggesting that CFM4 plays a role in growth and development of plants under normal growth conditions. In addition, cfm4 mutants showed retarded seed germination and seedling growth under stress conditions. No alteration in the splicing patterns of intron-containing chloroplast genes was observed in the mutant plants, but the processing of $16 \mathrm{~S}$ and $4.5 \mathrm{~S}$ rRNAs was abnormal in the mutant plants. Importantly, CFM4 was determined to possess RNA chaperone activity.
\end{abstract}

Conclusions: These results suggest that the chloroplast-targeted CFM4, one of two Arabidopsis genes encoding a single CRM domain-containing protein, harbors RNA chaperone activity and plays a role in the Arabidopsis growth and stress response by affecting rRNA processing in chloroplasts.

Keywords: Arabidopsis thaliana, Chloroplast, CRM domain, RNA-binding protein, RNA metabolism

\section{Background}

Chloroplasts are derived from cyanobacteria through endosymbiosis, and massive gene transfer from the plastid to the nucleus occurred during evolution [1]. Chloroplasts possess approximately 100-150 genes in their own circular genome that encodes messenger RNAs, ribosome RNAs, and transfer RNAs. In addition to its own gene products, functional communication between chloroplasts and nucleus is required, and many nuclear-encoded proteins are targeted to chloroplasts and play fundamental roles in the regulation of chloroplast gene expression. Expression of chloroplast genes is commonly regulated at posttranscriptional level, including mRNA processing, splicing, editing, decay, and translational control [2-5]. A sophisticated

\footnotetext{
* Correspondence: hskang@chonnam.ac.kr

'Department of Plant Biotechnology, College of Agriculture and Life Sciences, Chonnam National University, 300 Yongbong-dong, Buk-gu, Gwangju 500-757, Korea

Full list of author information is available at the end of the article
}

regulatory process between chloroplasts and nuclei is required to fine-tune chloroplast gene expression, and many nuclear-encoded RNA-binding proteins (RBPs) have been recently regarded as the primary elements that modulate posttranscriptional steps in chloroplasts $[2,4,6,7]$. Although chloroplasts share some features of RNA metabolism with their bacterial ancestors, chloroplasts require a more complicated mechanism of RNA metabolism compared to their ancestor, which have both prokaryotic and eukaryotic characteristics $[8,9]$. One particular example is the splicing of group I and group II introns. In contrast to self-splicing of prokaryotic introns, chloroplasts have lost their capacity to self-splice, and the splicing of group I and group II introns in chloroplasts requires many nuclear-encoded proteins that form protein complexes similar to spliceosomal complexes found in eukaryotes [10-14]. Therefore, involvement of nuclearencoded RBPs is indispensible for posttranscriptional 
regulation of RNA metabolism and gene expression in chloroplasts.

CRM (chloroplast RNA splicing and ribosome maturation) domain-containing proteins were first found in archaea and bacteria. Based on their structural data and predicted domain structures, CRM domain proteins were suggested to have RNA-binding activity [12]. Prokaryotes contain proteins harboring only a single CRM domain, whereas land plants contain proteins harboring multiple CRM domains. Splicing of group I and group II introns and tRNAs and processing of rRNAs in chloroplasts require different complexes of CRSs (for chloroplast RNA splicing), CAFs (for CRS2-associated factors), and CFMs (for CRM family members) that harbor multiple CRM domains [12,13,15-19]. It has also been demonstrated that mutation in CRM domain-containing protein genes results in pale-green phenotypes, delayed development, and aborted seed production in plants, indicating the important roles of CRM domain proteins in plant growth and development $[11,12,17,20,21]$.

Despite an increased understanding of the roles of CRM domain-containing proteins in chloroplast RNA metabolism and plant growth and development, the functional roles of most of the CRM domain-containing proteins have not been demonstrated experimentally. The Arabidopsis (Arabidopsis thaliana) and rice (Oryza sativa) genomes harbor the genes encoding 16 and 14 CRM domain-containing proteins, respectively [13]. Among the 16 Arabidopsis CRM domain-containing protein genes, two genes (At4g39040 and At2g21350) encode the smallest proteins harboring a single CRM domain [13]. However, the role of single CRM domain-containing proteins has not been demonstrated in plants. Here, we determined the developmental and stress response roles of a single CRM domain-containing protein (At4g39040). Because this protein belongs to subfamily group 4 among CRM domain proteins [13], we designated it as CRM family member subfamily4 (CFM4). We show that CFM4 possesses RNA chaperone activity and is involved in rRNA processing, which is important for normal growth, development, and the stress response in plants.

\section{Results}

\section{Structural features and characterization of CFM4 in Arabidopsis}

Sixteen predicted CRM domain family members occur in the Arabidopsis genome, and they are classified into four groups, such as CRS1 subfamily, CAF subfamily, subfamily 3 , and subfamily 4. Among the 16 CRM domain-containing protein genes, two genes (At4g39040 and At2g21350) encode proteins harboring a single CRM domain and are classified into subfamily group 4 [13]. We thus named At4g39040 as CFM4. The CFM4 protein contains a highly conserved GxxG sequence in the C-terminal half of the protein (Figure 1A and Additional file 1). The two single CRM domain-containing proteins (At4g39040 and At2g21350) share approximately 56\% amino acid sequence homology with each other. To examine whether the single CRM domain proteins are conserved in dicotyledonous and monocotyledonous plants, the amino acid sequences of single CRM domain proteins in diverse plant species, including Arabidopsis, Zea mays, Medicago truncatula, Vitis vinifera, Hordeum vulgare, Sorghum bicolor, and Oryza sativa, were compared. The results showed that CFM4 family proteins share $35-50 \%$ amino acid sequence homology among dicot and monocot plants and share $>70 \%$ amino acid sequence homology among monocot plants (Additional file 1), suggesting that the single CRM domain-containing proteins are functionally conserved in dicots and monocots.

The CRM proteins in Arabidopsis and rice have been predicted to be targeted mainly to chloroplasts or mitochondria. To determine the subcellular localization of CFM4, the cDNA encoding CFM4 was ligated in front of the green fluorescence protein (GFP) gene, and expression of the CFM4-GFP fusion protein was investigated in transgenic Arabidopsis plants. Strong GFP signals were observed in chloroplasts (Figure 1B). To examine whether CFM4 is also localized to mitochondria, Arabidopsis mitochondria were stained with Mito-tracker that is a red-fluorescent dye and stains mitochondria in live cells, and the signals from plastids in roots and chloroplasts in leaves were examined. The results showed that the signals from mitochondria did not overlap with the signals from chloroplasts, and GFP signals were observed exclusively in chloroplasts (Figure 1C and 1D). These results clearly indicate that CFM4 is localized to chloroplasts.

\section{CFM4 plays a role in Arabidopsis growth and senescence}

To determine the role of CFM4 during plant growth and development, the T-DNA insertion mutant lines in CFM4 (SALK_076439 and SALK_126978) were obtained, and their phenotypes were analyzed under normal and stress conditions. The absence of CFM4 expression in the knockout mutant lines was confirmed by RT-PCR analysis (Additional file 2). The wild-type and $c f m 4 \mathrm{mu}-$ tant plants were grown in MS medium or soil, and their phenotypes were observed during the entire life cycle (from germination to senescence) of the plants. Growth of the wild-type and mutant plants was not significantly different at 7 days after germination (DAG) (Additional file 3). However, growth of the plants was markedly different at later stages in that the size of the cfm4 mutants was much smaller than that of the wild-type plants at 20 or 23 DAG (Figure 2A and Additional file 3). The difference in flowering time between the wild-type and mutant plants was evident; cfm 4 mutants flowered 


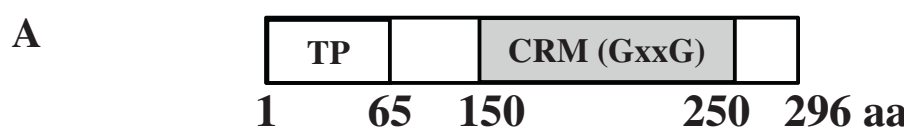

B
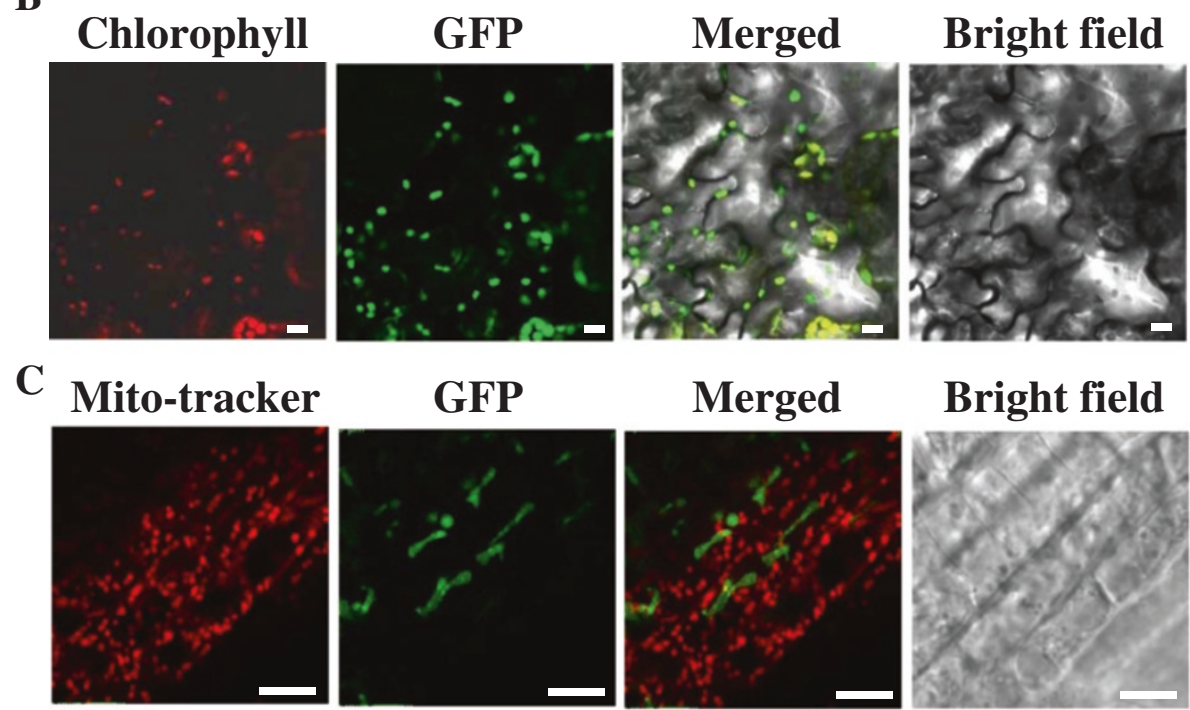

Merged

Bright field
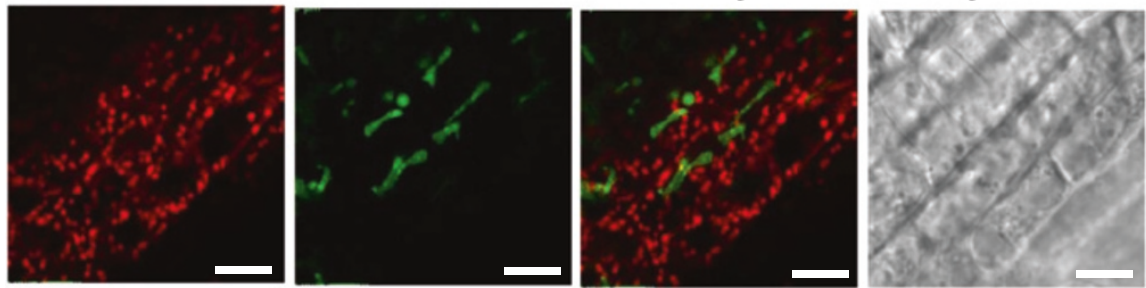

\section{Mito-tracker}
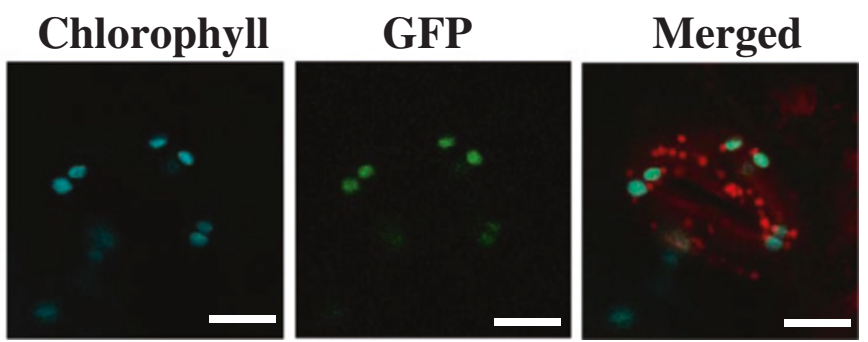

Figure 1 The domain structure and cellular localization of CFM4. (A) Schematic presentation of the domain structure of the CFM4 protein. The position of the CRM domain with a conserved GxxG sequence (gray box) is shown; TP, transit peptide. (B) Chloroplast localization of the CFM4 protein in Arabidopsis leaf. Red signals indicate chloroplast auto-fluorescence and green signals indicate GFP fluorescence. Bar $=10 \mu \mathrm{m}$ (C) Images showing mitochondria and GFP fluorescence in root. (D) Images showing mitochondria, chloroplast auto-fluorescence, and GFP fluorescence in leaf. Bar $=10 \mu \mathrm{m}$.

approximately 7 days later than the wild-type plants (Figure $2 \mathrm{~B}$ and Additional file 4). Although the $c f m 4$ mutants flowered much later than the wild-type plants, the size and number of leaves at the time of bolting were not different between the wild-type and mutant plants (Figure 2C), suggesting that CFM4 does not affect control of flowering time. No significant difference in plant height was observed between the wild-type and mutant plants at the time of maturity. The root growth of the cfm4 mutants was also retarded compared with that of the wild-type plants (Figure 2D and Additional file 5). To determine whether the retarded root growth was due to the decreases in cell size or cell number, the plasma membranes in the roots of the wild-type and cfm 4 mutant plants were stained with FM 4-64 dye and observed under confocal microscope. The results showed that the size of cells in cfm4 mutants was much smaller than that in the wild-type plants (Figure 2E). The retarded growth phenotypes of the mutant plants recovered to normal phenotypes in the complementation lines (Figure 2 and Additional files 4 and 5). All of these observations clearly demonstrate that CFM4 plays a role for normal growth of Arabidopsis plants. Because a recent study demonstrated that the growth retardation phenotypes of an Arabidopsis mutant are closely related to abscisic acid (ABA) biosynthesis and chloroplast RNA metabolism [22], we wanted to determine ABA levels in the wild-type plants, $c f m 4$ mutants, and complementation lines using an immunoassay method. The results showed that levels of ABA in cfm 4 mutants were approximately $70-80 \%$ of those in the wild-type plants (Figure 2F). We also analyzed transcript levels of the genes, including $A B A 1, A B A 2, A B A 3$, and NCED3 that are involved in ABA biosynthesis, and found that the levels of NCED3 were significantly lower in cfm4 mutants than in the wildtype plants (Figure 2G). These results suggest that the 

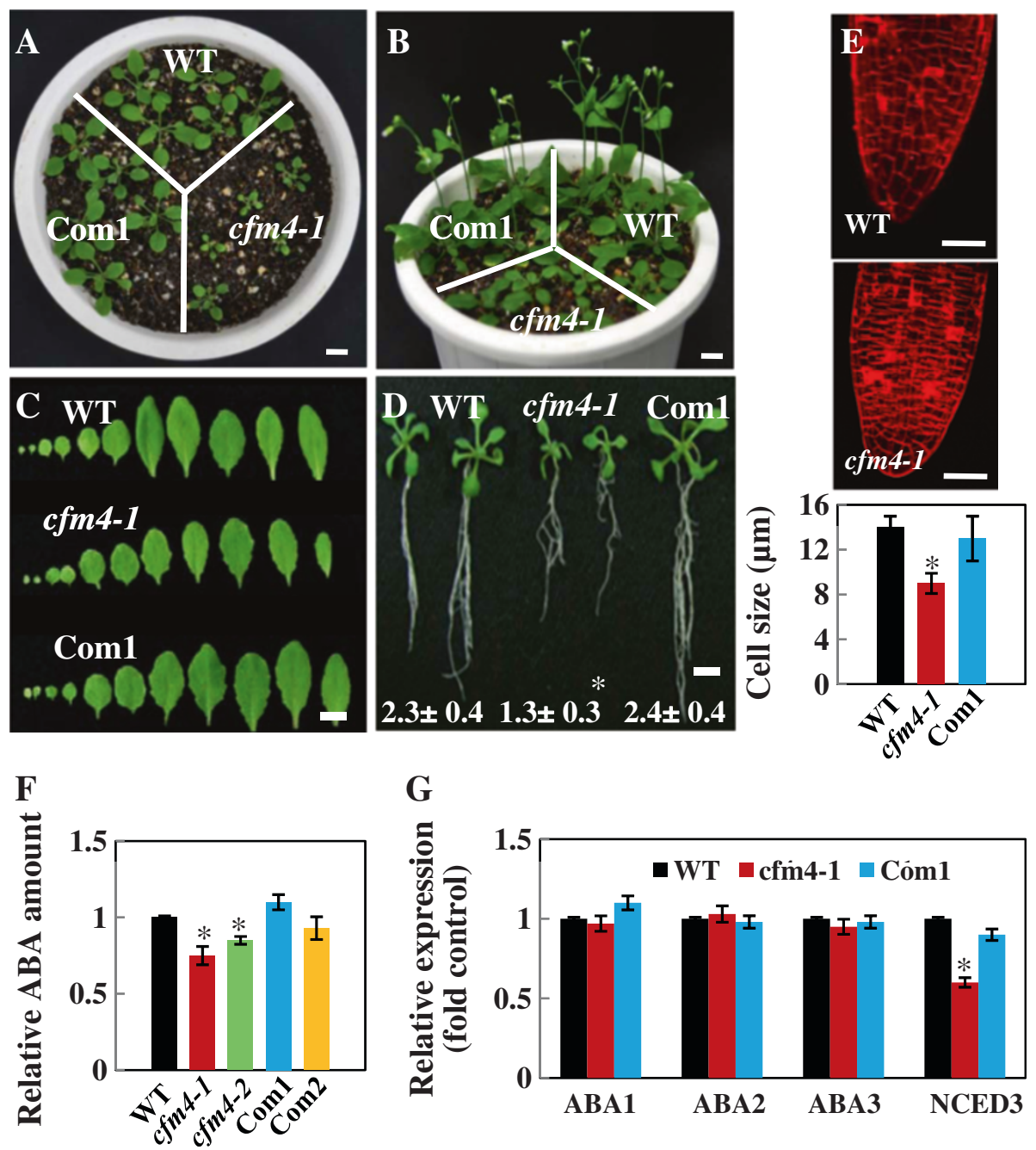

G

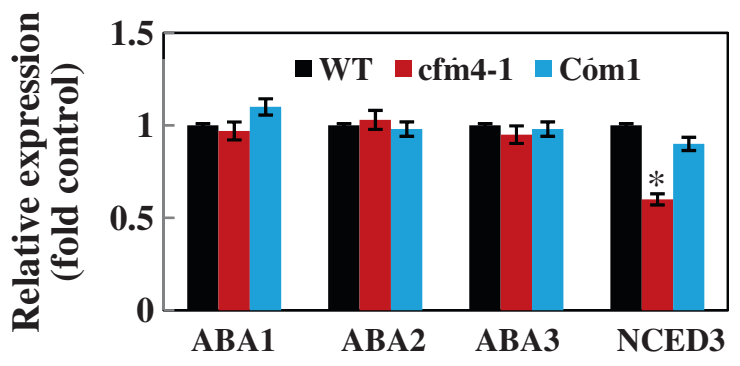

Figure 2 Phenotypes of $c \mathrm{fm} 4$ mutant plants and complementation lines. (A, B) Growth of the wild-type plants (WT), cfm4 mutants, and complementation line (Com1) at 20 and 33 days after germination (DAG). Scale bar $=1 \mathrm{~cm}$. (C) The size and number of leaves at emergence of floral buds on 26 DAG. Scale bar $=1 \mathrm{~cm}$. (D) Root growth $(\mathrm{cm})$ of the plants measured on 20 DAG. Scale bar $=1 \mathrm{~cm}$. (E) The size of cells in the roots of the plants measured on 26 DAG. Scale bar $=50 \mu \mathrm{m}$. (F) The amount of ABA was measured in 26-day-old plants, and relative amount to wild-type value is shown. (G) Transcript levels of ABA biosynthesis-related genes, ABA1, ABA2, ABA3, and NCED3, were measured in 26-day-old plants by real-time RT-PCR, and relative expression levels to wild-type value are shown. The values are mean \pm SE obtained from five independent experiments, and asterisk above the number indicates statistically different values between WT and mutant plants $(P \leq 0.05)$.

retarded growth phenotypes of $c f m 4$ mutants are related to impaired ABA biosynthesis.

With the observation that CFM4 plays a role in the growth of Arabidopsis, we subsequently examined whether CFM4 is involved in senescence. In the dark-induced senescence assay, it was evident that greening of the leaves of cfm 4 mutant plants was maintained for much longer compared with that of the wild-type plants when they were incubated under dark conditions (Figure 3A). Total chlorophyll (chlorophyll $\mathrm{a}+\mathrm{b}$ ) contents in cfm4 mutant plants were much higher than those in the wild-type plants (Figure 3B). Greening of the leaves of the complementation lines was similar to that of the wild-type plants, and the chlorophyll contents in the complementation lines were comparable with those in the wild-type plants (Figure 3). These results suggest that CFM4 plays a positive role in senescence.

\section{CFM4 is involved in Arabidopsis response to abiotic} stresses

To determine whether CFM4 plays a role in the plant response to environmental stresses, the wild-type, $c f m 4$ mutants, and complementation lines were grown in MS medium supplemented with $\mathrm{NaCl}$ for salt stress or with mannitol for dehydration stress, or the plants were grown at $10^{\circ} \mathrm{C}$ for cold stress treatment. We first compared seed germination rates of the plants under normal and stress conditions. No differences were observed in seed germination rates between the wild-type and mutant 

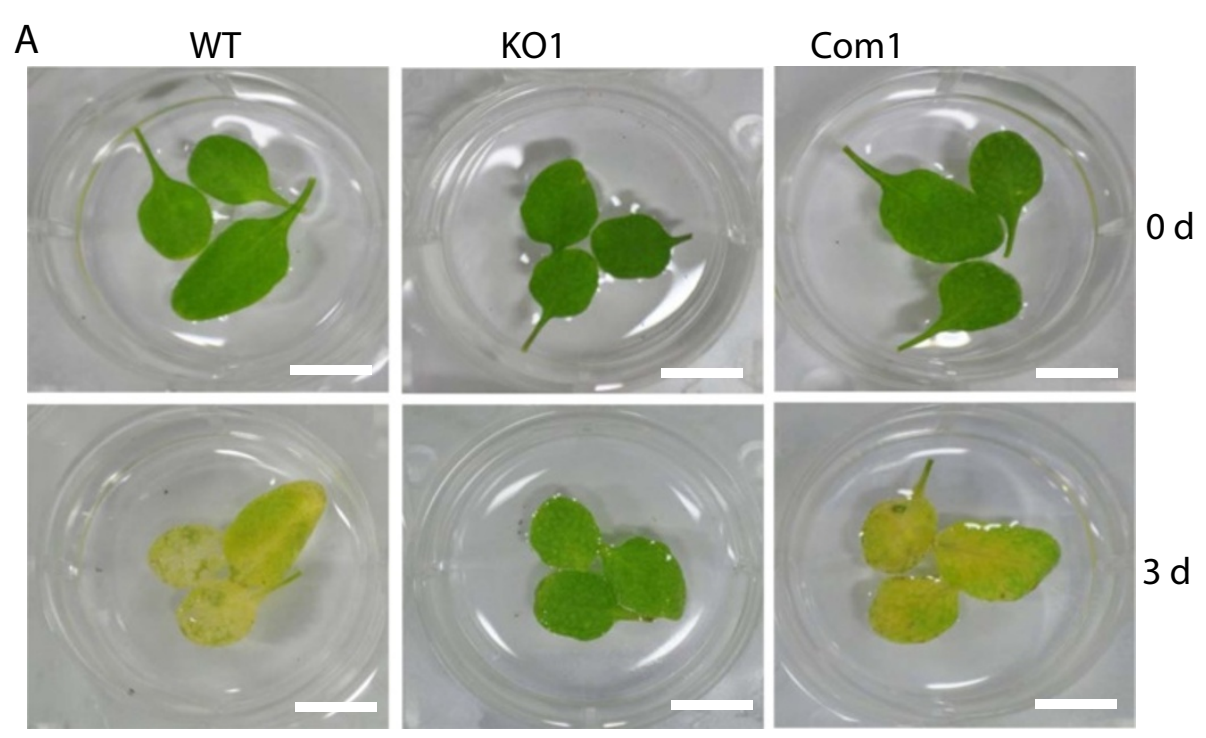

B

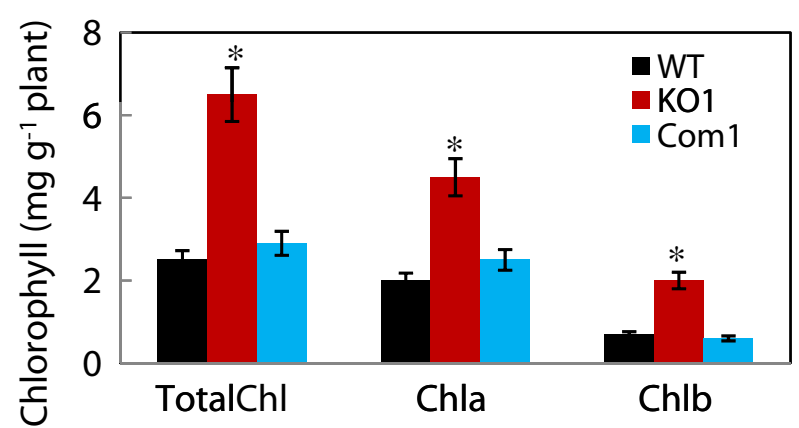

Figure 3 Delayed senescence of cfm4 mutant plants. (A) Rosette leaves from 4-week-old wild type (WT), cfm4 mutants, and complementation line (Com 1) were floated in water in the dark for 3 days. (B) Chlorophyll content was measured in the leaves of each plant 3 days after dark-induced senescence. The values are mean \pm SE obtained from three independent experiments, and asterisks above the columns indicate statistically different values between WT and mutant plants $(P \leq 0.05)$.

plants under normal conditions (Figure 4A). However, seed germination of $c f m 4$ mutants was retarded compared with that of the wild-type and complementation lines under salt or cold stress conditions (Figure 4B and 4C), whereas seed germination rates of all three genotypes were similar to each other under dehydration stress conditions (Additional file 6). Seedling growth of $c \mathrm{fm} 4$ mutants was also retarded compared with that of the wild-type and complementation lines under salt or cold stress (Figure 4). These results show that CFM4 affects seed germination and subsequent seedling growth of Arabidopsis under salt or cold stress conditions.

\section{CFM4 affects rRNA processing in chloroplasts}

The CRM domain-containing proteins have been demonstrated to be involved in the splicing of group II introns of chloroplast mRNAs and tRNAs $[13,16,18,19]$. Therefore, we first examined whether CFM4 is involved in the splicing of intron-containing genes in chloroplasts. Splicing patterns of all intron-containing chloroplast transcripts, including 15 mRNAs and 6 tRNAs, were analyzed by RT-PCR. The results showed that the splicing patterns of all intron-containing chloroplast transcripts were not altered in $c f m 4$ mutants compared with those in the wildtype plants (Figure 5A and Additional file 7), suggesting that CFM4 is not involved in intron splicing in chloroplasts. We next examined whether CFM4 is involved in rRNA processing. The levels of rRNAs, including 23S, $16 \mathrm{~S}, 5 \mathrm{~S}$, and $4.5 \mathrm{~S}$ rRNAs, in the wild type, cfm 4 mutant, and complementation line were determined by Northern blot analysis. To accurately determine relative levels of rRNA transcripts, Northern blot analysis was repeated four times, and the relative intensities of rRNA bands in cfm 4 mutants and complementation lines compared with those in wild type were calculated. The results showed that the transcript levels of the $23 \mathrm{~S}$ and $5 \mathrm{~S}$ rRNAs in $c f m 4$ 

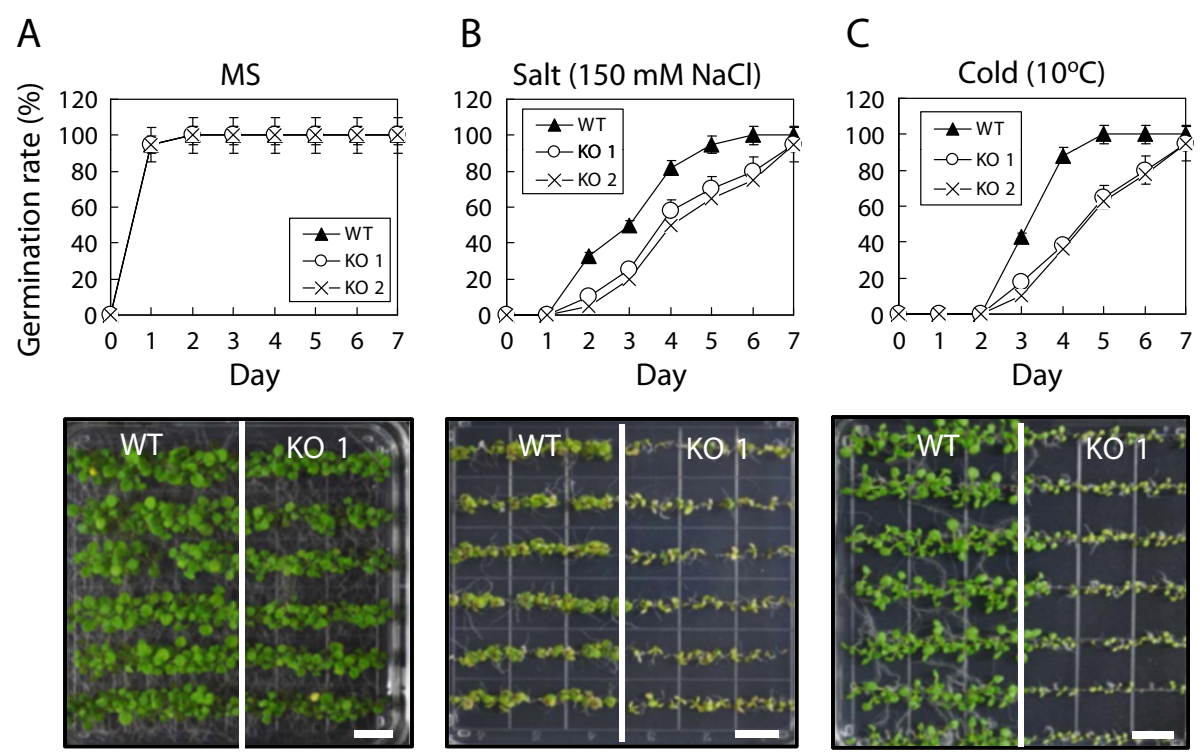

Figure 4 Response of $\mathrm{cfm} 4$ mutant plants to abiotic stresses. Seed germination and seedling growth of wild-type plant (WT) and cfm4 mutants (A) on MS medium, (B) on MS medium supplemented with $150 \mathrm{mM} \mathrm{NaCl}$, or (C) on MS medium at $10^{\circ} \mathrm{C}$. Germination rate was scored on the indicated days. The pictures were taken 16 days after germination. Scale bar $=1 \mathrm{~cm}$.

mutants were comparable with those in the wild-type plants. However, the mature-16S rRNA transcript levels in cfm4 mutant decreased compared with those in the wildtype plants (Figure $5 \mathrm{~B}$ ). In addition, the levels of mature4.5S rRNA decreased, whereas the levels of precursor- $4.5 \mathrm{~S}$ rRNA increased in cfm 4 mutant. These abnormal levels of $16 \mathrm{~S}$ and $4.5 \mathrm{~S}$ rRNAs observed in cfm4 mutants were restored to wild-type levels in the complementation line (Figure 5B). These results indicate that CFM4 affects the processing of $16 \mathrm{~S}$ and $4.5 \mathrm{~S}$ rRNAs.

\section{CFM4 possesses RNA chaperone activity}

RNA processing and intron splicing require proper folding of RNA substrates, and many RBPs that harbor RNA chaperone activity play an important role during these cellular processes [23-25]. Because CFM4 harbors a CRM domain that is known as an RNA binding module [13], we aimed to determine whether CFM4 possesses RNA chaperone activity. We first analyzed the complementation ability of CFM4 in the cold-sensitive E. coli BX04 mutant in which four cold shock proteins known as RNA chaperones are deficient and is high sensitivity to lower temperatures [26]. When the BX04 cells harboring each construct were incubated at $37^{\circ} \mathrm{C}$, all cells grew well with no difference. However, when the BX04. cells were exposed to cold shock at $20^{\circ} \mathrm{C}$, the cells expressing CFM4 or CspA as a positive control grew well at low temperatures, whereas the cells harboring the pINIII vector did not grow at low temperatures (Figure 6A). This result demonstrates that CFM4 has the ability to complement RNA chaperone-deficient E. coli mutant cells, suggesting that CFM4 functions as an RNA chaperone in E. coli under cold shock.

To further confirm whether CFM4 possesses RNA chaperone activity, the in vitro and in vivo nucleic acidmelting abilities of CFM4 were assessed via DNA-melting and transcription anti-termination assays. For the analysis of in vitro DNA-melting ability of CFM4, the recombinant GST-CFM4 fusion proteins were purified from E. coli (Additional file 8) and GST-CFM4 fusion protein was tested for its ability to destabilize base pairs in the synthetic DNA molecules labeled with a fluorophore (tetramethyl rhodamine) and quencher (dabcyl). Fluorescence of the molecular beacon increased after adding the GSTCFM4 proteins or the GST-CspA fusion proteins (positive control), confirming the DNA-melting activity of CFM4 (Figure 6B). By contrast, adding GST alone (negative control) did not increase fluorescence (Figure 6B). To determine whether CFM4 has the ability to destabilize base pairs in RNA, the in vivo RNA-melting ability of CFM4 was assessed via transcription anti-termination assays using E. coli RL211 cells that harbor a chloramphenicol resistance gene downstream from a trpL terminator with stem-loop structure [27]. When the RL211 cells were grown in the MS medium containing chloramphenicol, the cells expressing CFM4 or CspA grew well, suggesting that CFM4 and CspA destabilize the stem-loop structures present in the transcription termination signal. By contrast, the cells harboring the pINIII vector did not grow in chloramphenicol-containing MS medium (Figure 6C). Taken together, these results indicate that CFM4 possesses RNA chaperone activity. 

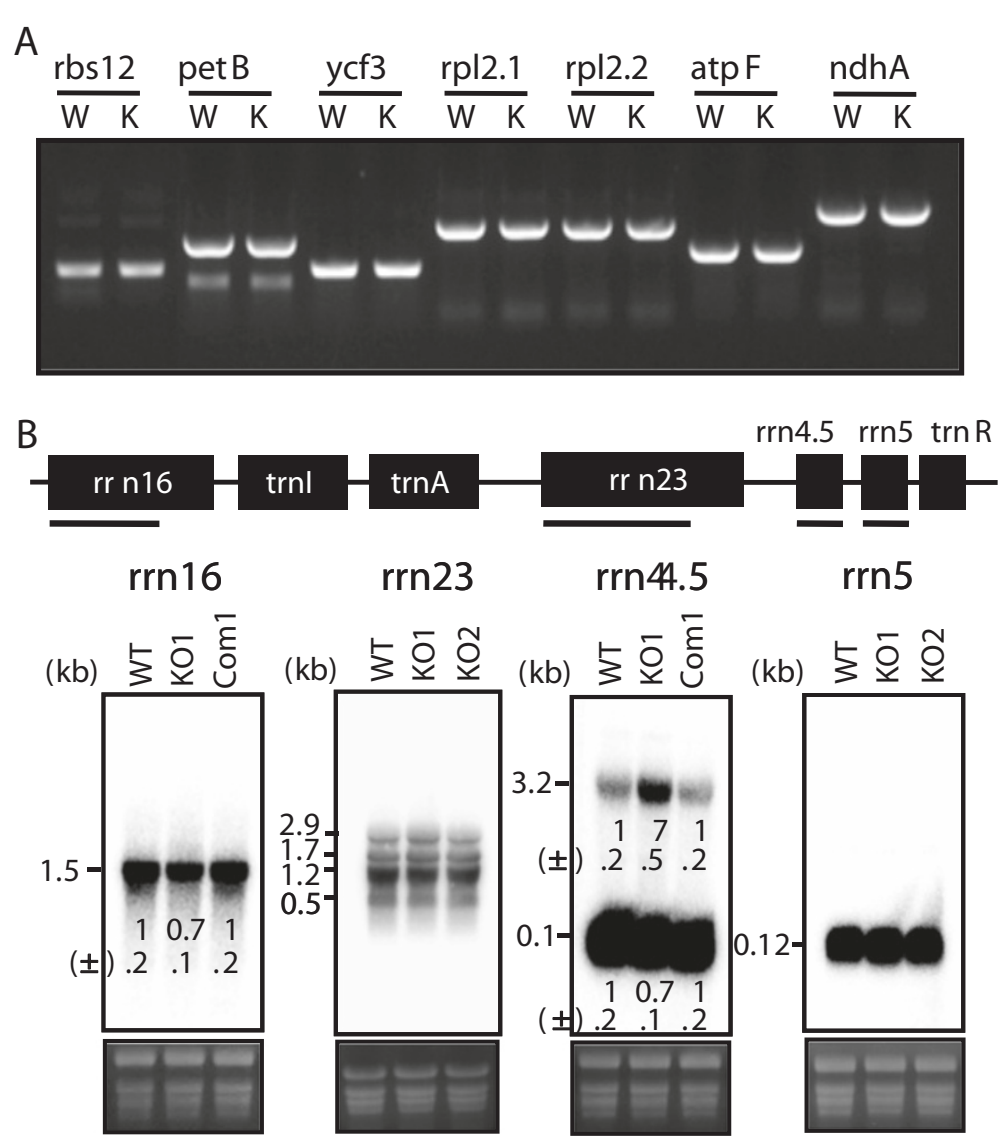

Figure 5 Splicing patterns of chloroplast transcripts and rRNA processing in cfm 4 mutant plants. (A) Total RNAs were extracted from 4-week-old wild-type (W) and cfm4 mutant (K), and the levels of intron-containing chloroplast transcripts were analyzed by RT-PCR. Identical results were obtained from independent experiments, and a representative result is shown. (B) Total RNA was extracted from 4-week-old wild-type (WT), cfm4 mutants, and complementation line (Com1) and was separated on a 1.2\% formaldehyde agarose gel. The levels of the processed products of 23S, 16S, 5S, and 4.5S rRNAs were determined by Northern blot analysis using the probes corresponding to each gene, represented by thick lines below each gene. The relative intensities of rRNA bands in cfm4 mutants and complementation lines compared with those in wild type were calculated and values under each lane are means $\pm S D(n=4)$.

\section{Discussion}

The results presented here demonstrate that CFM4, one of two Arabidopsis genes encoding single CRM domaincontaining protein, plays a role in the Arabidopsis growth and stress response by affecting rRNA processing in chloroplasts. Analysis of the T-DNA knockout lines indicated that CFM4 exhibits important function during plant growth and senescence (Figures 2 and 3), which is in agreement with its postulated roles in chloroplast RNA metabolism. Our current results point to the important roles of CRM domain-containing proteins in plant growth and development. It has been demonstrated that mutation in the genes encoding multiple CRM domain-containing proteins causes pale-green phenotypes, delayed development, and aborted seed production in plants $[11,12,17,20,21]$. In addition to its roles in the growth and senescence of Arabidopsis under normal growth conditions, CFM4 also affected the plant response to abiotic stresses. Seed germination and seedling growth of cfm4 mutants were retarded compared with those of the wild-type plants under salt or cold stress conditions (Figure 4), suggesting that CFM4 plays a positive role in seed germination and seedling growth of Arabidopsis under salt or cold stress conditions. Interestingly, the effect of CFM4 on seed germination and seedling growth of plants was confined to salt or cold stress, as no differences in seed germination and seedling growth were observed between the wild type and cfm 4 mutants under dehydration stress conditions. Notably, the genes encoding single CRM domain-containing proteins as well as the genes encoding multiple CRM domain-containing proteins play important roles in plant growth, development, and stress response.

All multiple CRM domain-containing proteins whose functions have been characterized in plants are involved in the splicing of chloroplast group II and I introns [17]. CAF1, CAF2, and CRS1, the three maize proteins harboring multiple copies of the domain, are required for 


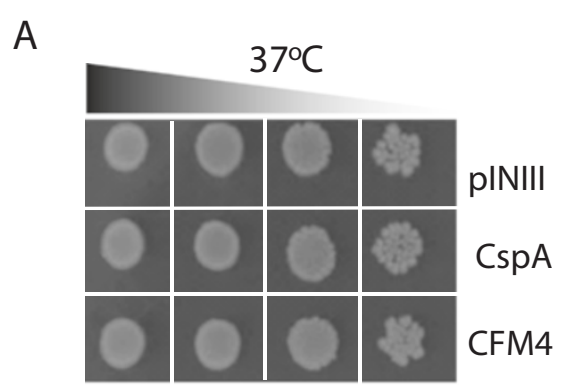

B

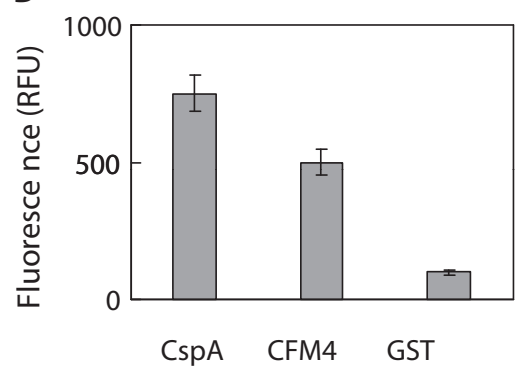

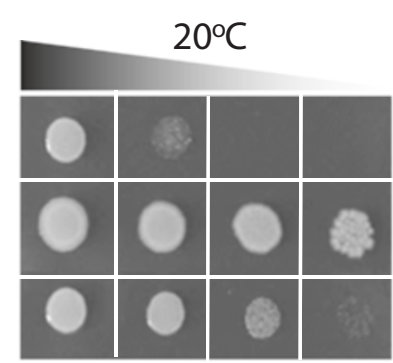

C

PINIII CspA CFM4

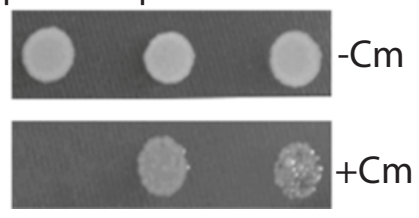

Figure 6 RNA chaperone activity of the CFM4 protein. (A) Complementation ability of CFM4 in E. coli BX04 mutant cells during cold shock. The diluted cultures of E. coli cells expressing CFM4, CspA (positive control), or pINIII vector (negative control) were spotted on LB-agar plates, and the cells were incubated at either $37^{\circ} \mathrm{C}$ or $20^{\circ} \mathrm{C}$. (B) DNA-melting ability of CFM4. Fluorescence of the molecular beacons was measured after adding the recombinant GST-CFM4, GST-CspA (positive control), or GST (negative control) proteins. (C) Transcription anti-termination activity of CFM4. The E. coli RL211 cells expressing each construct were spotted on LB-agar medium with (+) or without (-) chloramphenicol (Cm), and the cells were incubated at $37^{\circ} \mathrm{C}$.

the splicing of group II introns in chloroplasts [15,28]. In addition, it has been demonstrated that CFM2 and CFM3 together with CRS2/CAF complexes promote the splicing of chloroplast introns [12,17]. In contrast to the well-characterized roles of multiple CRM domaincontaining proteins in the splicing of chloroplast introns, CFM4 plays no role in the splicing of chloroplast introns (Figure 5A and Additional file 7). However, our current analysis shows that CFM4 is involved in the processing and maturation of $16 \mathrm{~S}$ and $4.5 \mathrm{~S}$ rRNAs (Figure $5 \mathrm{~B}$ ). This observation is in line with previous findings that prokaryotic proteins harboring a single CRM domain participate in ribosome maturation [13]. The YhbY, the CRM domain protein in Escherichia coli, is a small protein with molecular mass of $\sim 10 \mathrm{kDa}$ and is associated with pre-50S ribosomal subunits, which function in ribosome assembly [13]. Among the 16 CRM domain proteins found in Arabidopsis, CRM4 is the most homologous to YhbY in that it harbors little else except a single CRM domain (Additional file 1). We propose that the delayed growth of $c f m 4 \mathrm{mu}-$ tants is due to, at least in part, improper processing of $16 \mathrm{~S}$ and $4.5 \mathrm{~S}$ rRNAs in chloroplasts. Interestingly, improper rRNA processing in $\mathrm{cfm} 4$ mutants resulted in impaired ABA biosynthesis (Figure 2), which supports a notion that proper chloroplast function is required not only for active photosynthesis but also for ABA biosynthesis, both of which are required for normal plant growth [22].
The proposed mechanistic role of CFM4 as an RNA chaperone in chloroplast rRNA metabolism is intriguing. RNA processing as well as intron splicing requires proper folding of RNA substrates, and many RBPs play an important role in RNA-RNA and RNA-protein interactions during RNA metabolism [23,29-31]. RNA chaperones are nonspecific RBPs that bind diverse RNA substrates and help RNAs fold by inducing structural rearrangement of misfolded RNAs $[25,32,33]$. It has recently been demonstrated that one of the minor spliceosomal proteins U11/ U12-31 K possesses RNA chaperone activity and is indispensible for correct intron splicing and normal growth and development in Arabidopsis and rice [23,34], which emphasizes the important role of RNA chaperones in maintaining RNA substrates in splicing-competent structures for correct processing to occur. Involvement of RNA chaperone activity in the splicing of group I and group II introns has been demonstrated in yeast mitochondria. The yeast DEAD-box protein MSS116 promotes the splicing of both group I and group II introns in mitochondria via functioning as an RNA chaperone [35-38]. Our current analysis clearly indicates that CFM4 harbors RNA chaperone activity (Figure 6). It is likely that RNA chaperone activity of CFM4 is needed to maintain precursor-rRNA in processing-competent structures for subsequence rRNA processing. Although it is not clear at present how CFM4 affects processing of only two (16S and 4.5S) rRNAs out of 
the four rRNAs in the chloroplast, it is possible that CFM4 recognizes specific sequence or structural elements in $16 \mathrm{~S}$ and $4.5 \mathrm{~S}$ rRNAs and the RNA chaperone activity of CFM4 is involved in the formation of processing-competent structures of these rRNAs.

\section{Conclusions}

The present study shows that CFM4 harboring RNA chaperone activity is involved in rRNA processing in chloroplasts, which is important for growth and the stress response of plants. Although much progress has been made in the characterization of the roles of CRM domain-containing proteins in intron splicing and rRNA processing during chloroplast gene expression, the roles of many CRM proteins in plant growth and development as well as chloroplast RNA metabolism remain largely unknown. In particular, understanding the biological function of single CRM domain-containing proteins is far behind compared with that of multiple CRM domaincontaining proteins. Exploring the importance of the number of CRM domains and involvement of each CRM domain in substrate recognition during intron splicing and rRNA processing should provide a much deeper insight into the mechanistic role of CRM family members in chloroplast RNA metabolism.

\section{Methods}

\section{Plant materials and growth conditions}

The Arabidopsis thaliana used in this study was Col-0 ecotype. The plants were grown either in a mixture of vermiculite, peat moss, and perlite or on half-strength Murasige \& Skoog (MS) medium containing 1\% sucrose at $23 \pm 2^{\circ} \mathrm{C}$ under long-day conditions (16 h light $/ 8 \mathrm{~h}$ dark cycle). To construct overexpressing transgenic plants and complementation lines, the CFM4 cDNA coding sequence was cloned into the NcoI/BstEII of pCambia1301 vector containing the cauliflower mosaic virus $35 \mathrm{~S}$ promoter. The Arabidopsis transformation was carried out via vacuum infiltration [39] using Agrobacteruim tumefaciens GV3101. The $\mathrm{T}_{3}$ transgenic lines were selected and subsequently utilized for the phenotype investigation. Expression of CFM4 in each transgenic plant was analyzed by RT-PCR using the gene-specific primers listed in Additional file 9. Arabidopsis mutant seeds with T-DNA inserted into the CFM4 gene (SALK_076439 and SALK_ 126978) were obtained from The Arabidopsis Biological Resource Center (Columbus, OH, USA). The absence of CFM4 expression in the mutant lines was confirmed by RT-PCR using the gene-specific primers listed in Additional file 9.

\section{Analysis of cellular localization of CFM4 in Arabidopsis} To determine the cellular localization of CFM4, the cDNA corresponding to the full-length CFM4 was ligated using the XbaI/BamHI site in front of the GFP gene, and CFM4-GFP fusion proteins were expressed under the control of the CaMV $35 \mathrm{~S}$ promoter in Arabidopsis. The GFP signals in the leaves and roots of the plants were observed using a Zeiss LSM510 laser scanning confocal microscope (Carl Zeiss Inc. Thornwoold, NY, USA). The excitation and emission wavelengths were 488 and $545 \mathrm{~nm}$, respectively. Mitochondria in the leaves and roots of the plants were stained with MitoTracker ${ }^{\oplus}$ Red CMXRos (Invitrogen).

\section{RNA extraction and RT-PCR}

Total RNAs were extracted from frozen tissues using the Plant RNeasy Extraction kit (Qiagen, Valencia, CA, USA), and the concentration of RNAs was quantified by spectrophotomety. RT-PCR was performed using the primers spanning the T-DNA insertion site to confirm whether the T-DNA tagged mutant seeds from ABRC were knockout mutants. To examine splicing patterns of intron-containing genes, total RNAs were extracted from 4-week-old plants, and $100 \mathrm{ng}$ RNA was used for RT-PCR with the gene-specific primers listed in Additional file 9. To examine the expression levels of ABA biosynthesis-related genes, total RNAs were extracted from 26-day-old plants, and 100 ng RNA was used for quantitative real-time RT-PCR with the genespecific primers listed in Additional file 9.

\section{Measurement of ABA content}

Ten grams of plant tissues at 26 DAG were ground in liquid nitrogen, mixed with an extraction solution $(80 \%$ methanol, $2 \%$ acetic acid), and incubated overnight at $4^{\circ} \mathrm{C}$. After centrifugation at $2000 \times \mathrm{g}$ for $10 \mathrm{~min}$, the pellet was dissolved in $10 \%$ methanol and TBS (50 mM Tris, $0.1 \mathrm{mM} \mathrm{MgCl}_{2}$, and $0.15 \mathrm{M} \mathrm{NaCl}, \mathrm{pH} \mathrm{7.8).} \mathrm{ABA}$ concentration was determined using Phytodetek ${ }^{\ominus}$ ABA Test kit (Agdia Inc., Elkhart, Indiana, USA) according to the manufacturer's instructions.

\section{Northern blot analysis of rRNA transcripts in chloroplasts}

Total RNA was extracted from 4-week-old wild-type and cfm4 mutant plants for chloroplast rRNA processing analysis. Five or ten micrograms of total RNA was electrophoresed on an $1.2 \%$ agarose or $16.5 \%$ formaldehyde $(\mathrm{w} / \mathrm{v})$ gel, blotted to a Hybond- $\mathrm{N}^{+}$nylon membrane (Amersham Biosciences, Parsippany, NJ, USA), and then cross-linked under UV. Hybridization with ${ }^{32} \mathrm{P}$-labeled probes was performed in $0.15 \%$ SDS, $5 \times$ SSC, $5 \times$ Denhardt's solution, salmon sperm DNA (10 mg/ml) and $50 \%$ formamide at $42^{\circ} \mathrm{C}$ overnight. The membrane was washed $2-3$ times in $2 \times$ SSC and $0.1 \%$ SDS at room temperature and once in $0.1 \times \mathrm{SSC}$ and $0.1 \%$ SDS at $42^{\circ} \mathrm{C}$. The genes encoding chloroplast rRNAs were cloned into the pGEM-T Easy vector using the Arabidopsis chloroplast sequence information 
(GenBank accession no. AP00023), and the hybridization probes were amplified by PCR from the chloroplast rDNAcloned vector. The probes contained rDNA sequences of the following region: rrn16S (position 101,500-102,482), rrn23S-5' (position 104,691-106,691), rrn23S-3' (position 106,692-107,500), rrn4.5S (position 107,599-107,701), and rrn5S (130,580-130,700). The probes were labeled with ${ }^{32} \mathrm{P}$ dCTP using the Random Primer DNA Labeling Kit (TaKaRa Bio., Shiga, Japan) according to the manufacturer's instructions.

\section{RNA chaperone assay}

For the cold shock and transcription anti-termination assays in E. coli, the pINIII vector expressing CFM4 was constructed essentially as described previously [40]. In the cold shock assay, the vector was introduced into E. coli BX04 mutant cells [26] that lack four cold shock proteins and thus are highly sensitive to cold stress. The BX04 mutant cells harboring either pINIII-CFM4, pINIII-CspA (positive control), or pINIII (negative control) were grown in Luria-Bertani (LB) medium containing ampicillin and kanamycin, and the serial-diluted cultures (from $10^{-1}$ to $10^{-5}$ ) of BX04 cells were spotted on LB medium and incubated at low temperature $\left(20^{\circ} \mathrm{C}\right)$. In the transcription antitermination assay, E. coli RL211 cells [27] transformed with each construct were grown in liquid LB medium and spotted on LB-carbenicillin plates with or without chloramphenicol. Growth of the cells was inspected on a daily basis.

For in vitro DNA-melting assay, the DNA molecules labeled with a fluorophore (tetramethyl rhodamine) and quencher (dabcyl) were synthesized as previously described $[40,41]$ For the expression and purification of recombinant GST-CFM4 fusion proteins in E. coli, the coding region of CFM4 was cloned into pGEX-5X-2 vector (Amersham Pharmacia Biosciences). The recombinant GST-CFM4 fusion proteins were expressed in BL21 DE3 competent cells (Promega) and purified using glutathione Sepharose 4B resin. The fluorescence emitted from the reaction between the GST-CFM4 fusion proteins and the DNA molecules was measured using a Spectra Max GeminiXS spectrofluorometer (Molecular Devices, Sunnyvale, CA, USA) with excitation and emission wavelengths of $555 \mathrm{~nm}$ and $575 \mathrm{~nm}$, respectively.

\section{Additional files}

Additional file 1: Alignment of the amino acid sequences of a single CRM domain-containing proteins from various plant species.

Additional file 2: Confirmation of knockout mutants and complementation lines.

Additional file 3: Phenotypes of cfm4 mutant plants.

Additional file 4: Phenotypes of cfm4 mutant plant and complementation line.
Additional file 5: Root growth of cfm4 mutant and complementation lines.

Additional file 6: Response of cfm4 mutant plants to dehydration stress.

Additional file 7: Splicing patterns of chloroplast transcripts in cfm4 mutant plant.

Additional file 8: Purification of recombinant glutathione S-transferase CFM4 fusion protein in E. coli.

Additional file 9: Table S1. Gene-specific primer pairs used in RT-PCR experiments.

\section{Abbreviations}

CRM: Chloroplast RNA splicing and ribosome maturation; CFM4: CRM family member subfamily 4; DAG: Day after germination; RBP: RNA-binding protein.

\section{Competing interests}

The authors declare that they have no competing interests.

\section{Authors' contribution}

$\mathrm{KL}$ and $\mathrm{HK}$ designed the experiments; $\mathrm{KL}, \mathrm{HJL}$, DHK and $\mathrm{YJ}$ conducted most of research and analyzed the data together with HSP and HK; KL and HK contributed to the writing of the manuscript. All authors read and approved the final manuscript.

\section{Acknowledgements}

We thank Drs. M. Inouye and S. Phadtare for the BX04 mutant cells and the pINIII vector and Dr. R. Landick for the E. coli RL211 cells. This study was supported by grants from the Mid-career Researcher Program through a National Research Foundation of Korea grant funded by the Ministry of Education, Science and Technology (2011-0017357) and from the Next-Generation BioGreen21 Program (PJ00949102), Rural Development Administration, Republic of Korea.

\section{Author details}

${ }^{1}$ Department of Plant Biotechnology, College of Agriculture and Life Sciences, Chonnam National University, 300 Yongbong-dong, Buk-gu, Gwangju 500-757, Korea. '2Department of Systems Biology, Yonsei University, Seoul 120-749, Korea.

Received: 21 December 2013 Accepted: 11 April 2014 Published: 16 April 2014

\section{References}

1. Myouga F, Akiyama K, Motohashi R, Kuromori T, Ito T, lizumi H, Ryusui R, Sakurai T, Shinozaki K: The Chloroplast Function Database: a large-scale collection of Arabidopsis Ds/Spm- or T-DNA-tagged homozygous lines for nuclear-encoded chloroplast proteins, and their systematic phenotype analysis. Plant J 2010, 61:529-542.

2. Marin-Navarro J, Manuell AL, Wu J, Mayfield PS: Chloroplast translation regulation. Photosynth Res 2007, 94:359-374.

3. de Longevialle AF, Small ID, Lurin C: Nuclearly encoded splicing factors implicated in RNA splicing in higher plant organelles. Mol Plant 2010, 3:691-705.

4. Stern DB, Goldschmidt-Clermont M, Hanson MR: Chloroplast RNA Metabolism. Annu Rev Plant Biol 2010, 61:125-155.

5. Barkan A: Expression of plastid genes: organelle-specific elaborations on a prokaryotic scaffold. Plant Physiol 2011, 155:1520-1532.

6. Danon A: Translational regulation in the chloroplast. Plant Physiol 1997, 115:1293-1298.

7. Nickelsen J: Chloroplast RNA-binding proteins. Curr Genet 2003, 43:392-399.

8. Schmitz-Linneweber C, Barkan A: RNA splicing and RNA editing in chloroplasts, Cell and Molecular Biology of Plastids. Berlin Heidelberg: Springer; 2007:213-248.

9. Stoppel R, Meurer J: Complex RNA metabolism in the chloroplast: an update on the psbB operon. Planta 2013, 237:441-449.

10. Jenkins $B D$, Kulhanek DJ, Barkan A: Nuclear mutations that block group II RNA splicing in maize chloroplasts reveal several intron classes with distinct requirements for splicing factors. Plant Cell 1997, 9:283-296. 
11. Asakura Y, Barkan A: Arabidopsis orthologs of maize chloroplast splicing factors promote splicing of orthologous and species-specific group II introns. Plant Physiol 2006, 142:1656-1663.

12. Asakura $Y$, Barkan A: A CRM domain protein functions dually in group I and group II intron splicing in land plant chloroplasts. Plant Cell 2007, 19:3864-3875.

13. Barkan A, Klipcan L, Ostersetzer O, Kawamura T, Asakura Y, Watkins KP: The CRM domain: an RNA binding module derived from an ancient ribosome-associated protein. RNA 2007, 13:55-64.

14. Bryant N, Lloyd J, Sweeney C, Myouga F, Meinke D: Identification of nuclear genes encoding chloroplast-localized proteins required for embryo development in Arabidopsis. Plant Physiol 2011, 155:1678-1689.

15. Till B, Schmitz-Linneweber C, Williams-Carrier R, Barkan A: CRS1 is a novel group II intron splicing factor that was derived from a domain of ancient origin. RNA 2001, 7:1227-1238

16. Ostersetzer O, Cooke AM, Watkins KP, Barkan A: CRS1, a chloroplast group II intron splicing factor, promotes intron folding through specific interactions with two intron domains. Plant Cell 2005, 17:241-255.

17. Asakura Y, Bayraktar OA, Barkan A: Two CRM protein subfamilies cooperate in the splicing of group IIB introns in chloroplasts. RNA 2008, 14:2319-2332.

18. Keren I, Klipcan L, Bezawork-Geleta A, Kolton M, Shaya F, Ostersetzer-Biran O: Characterization of the molecular basis of group II intron RNA recognition by CRS1-CRM domains. J Biol Chem 2008, 283:23333-23342.

19. Kroeger TS, Watkins KP, Friso G, van Wijk KJ, Barkan A: A plant-specific RNA-binding domain revealed through analysis of chloroplast group II intron splicing. Proc Natl Acad Sci U S A 2009, 106:4537-4542.

20. Stern DB, Hanson MR, Barkan A: Genetics and genomics of chloroplast biogenesis: maize as a model system. Trends Plant Sci 2004, 9:293-301.

21. Zmudjak M, Colas des Francs-Small C, Keren I, Shaya F, Belausov E, Small I, Ostersetzer-Biran O: mCSF1, a nucleus-encoded CRM protein required for the processing of many mitochondrial introns, is involved in the biogenesis of respiratory complexes I and IV in Arabidopsis. New Phytol 2013, 199:379-394.

22. Lee K-H, Park J, Williams DS, Xiong Y, Hwang I, Kang B-H: Defective chloroplast development inhibits maintenance of normal levels of abscisic acid in a mutant of the Arabidopsis $R H 3$ DEAD-box protein during early post-germination growth. Plant J 2013, 73:720-732.

23. Kim WY, Jung HJ, Kwak KJ, Kim MK, Oh SH, Han YS, Kang H: The Arabidopsis U12-type spliceosomal protein U11/U12-31K is involved in U12 intron splicing via RNA chaperone activity and affects plant development. Plant Cell 2010, 22:3951-3962.

24. Jung HJ, Park SJ, Kang H: Regulation of RNA Metabolism in Plant Development and Stress Responses. J Plant Biol 2013, 56:123-129.

25. Kang H, Park SJ, Kwak KJ: Plant RNA chaperones in stress response. Trends Plant Sci 2013, 18:100-106.

26. Xia $\mathrm{B}, \mathrm{Ke} \mathrm{H}$, Inouye M: Acquirement of cold sensitivity by quadruple deletion of the cspA family and its suppression by PNPase S1 domain in Escherichia coli. Mol Microbiol 2001, 40:179-188.

27. Landick R, Stewart J, Lee DN: Amino acid changes in conserved regions of the beta-subunit of Escherichia coli RNA polymerase alter transcription pausing and termination. Genes Dev 1990, 4:1623-1636.

28. Ostheimer GJ, Williams-Carrier R, Belcher S, Osborne E, Gierke J, Barkan A: Group II intron splicing factors derived by diversification of an ancient RNA-binding domain. EMBO J 2003, 22:3919-3929.

29. Staley JP, Guthrie C: Mechanical devices of the spliceosome: motors, clocks, springs, and things. Cell 1998, 92:315-326.

30. Prikryl J, Watkins KP, Friso G, van Wijk KJ, Barkan A: A member of the Whirly family is a multifunctional RNA- and DNA-binding protein that is essential for chloroplast biogenesis. Nucleic Acids Res 2008, 36:5152-5165.

31. Lorkovic ZJ: Role of plant RNA-binding proteins in development, stress response and genome organization. Trends Plant Sci 2009, 14:229-236.

32. Herschlag D: RNA chaperones and the RNA folding problem. J Biol Chem 1995, 270:20871-20874.

33. Rajkowitsch L, Chen D, Stampfl S, Semrad K, Waldsich C, Mayer O, Jantsch MF, Konrat R, Blasi U, Schroeder R: RNA chaperones, RNA annealers and RNA helicases. RNA Biol 2007, 4:118-130.

34. Kwak KJ, Park SJ, Han JH, Kim MK, Oh SH, Han YS, Kang H: Structural determinants crucial to the RNA chaperone activity of glycine-rich RNA-binding proteins 4 and 7 in Arabidopsis thaliana during the cold adaptation process. J Exp Bot 2011, 62:4003-4011.
35. Mohr S, Stryker JM, Lambowitz AM: A DEAD-box protein functions as an ATP-dependent RNA chaperone in group I intron splicing. Cell 2002, 109:769-779.

36. Huang HR, Rowe CE, Mohr S, Jiang Y, Lambowitz AM, Perlman PS: The splicing of yeast mitochondrial group I and group II introns requires a DEAD-box protein with RNA chaperone function. Proc Natl Acad Sci U S A 2005, 102:163-168.

37. Mohr S, Matsuura M, Perlman PS, Lambowitz AM: A DEAD-box protein alone promotes group II intron splicing and reverse splicing by acting as an RNA chaperone. Proc Natl Acad Sci U S A 2006, 103:3569-3574.

38. Halls C, Mohr S, Del Campo M, Yang Q, Jankowsky E, Lambowitz AM: Involvement of DEAD-box proteins in group I and group II intron splicing. Biochemical characterization of Mss116p, ATP hydrolysis-dependent and -independent mechanisms, and general RNA chaperone activity. J Mol Biol 2007, 365:835-855.

39. Bechtold N, Pelletier G: In planta Agrobacterium-mediated transformation of adult Arabidopsis thaliana plants by vacuum infiltration. Methods $\mathrm{Mol}$ Biol 1998, 82:259-266.

40. Kim JS, Park SJ, Kwak KJ, Kim YO, Kim JY, Song J, Jang B, Jung CH, Kang H: Cold shock domain proteins and glycine-rich RNA-binding proteins from Arabidopsis thaliana can promote the cold adaptation process in Escherichia coli. Nucleic Acids Res 2007, 35:506-516.

41. Phadtare $S$, Inouye $M$, Severinov K: The nucleic acid melting activity of Escherichia coli $\mathrm{CspE}$ is critical for transcription antitermination and cold acclimation of cells. J Biol Chem 2002, 277:7239-7245.

doi:10.1186/1471-2229-14-98

Cite this article as: Lee et al:: A nuclear-encoded chloroplast protein harboring a single CRM domain plays an important role in the Arabidopsis growth and stress response. BMC Plant Biology 2014 14:98.

\section{Submit your next manuscript to BioMed Central and take full advantage of:}

- Convenient online submission

- Thorough peer review

- No space constraints or color figure charges

- Immediate publication on acceptance

- Inclusion in PubMed, CAS, Scopus and Google Scholar

- Research which is freely available for redistribution 